\title{
APPLICATION OF A LEMMA ON BILINEAR FORMS TO A PROBLEM IN NONLINEAR OSCILLATIONS
}

\author{
A. C. LAZER
}

\begin{abstract}
In this note we give a simple condition for nondegeneracy of symmetric bilinear forms on infinite dimensional vector spaces. We apply this condition and elementary properties of Fourier series to prove a uniqueness theorem for periodic solutions of a class of second order nonlinear differential systems.
\end{abstract}

In [5] the present author and D. A. Sánchez considered the differential equation

$$
x^{\prime \prime}+\operatorname{grad} G(x)=p(t)=p(t+2 \pi)
$$

where $p \in C\left(R, R^{n}\right), G \in C^{2}\left(R^{n}, R\right)$. The equation may be considered as the Newtonian equations of motion of a mechanical system subject to conservative internal forces and periodic exciting forces. In [5] it was shown that if there exists an integer $N$ and numbers $\mu_{N}$ and $\mu_{V^{*+1}}$ such that

$$
N^{2}<\mu_{N} \leqq \mu_{N+1}<(N+1)^{2},
$$

and for all $a \in R^{n}$,

$$
\mu_{N^{\prime}} I \leqq\left(\partial^{2} G(a) / \partial x_{i} \partial x_{j}\right) \leqq \mu_{N+1} l
$$

where $I$ is the $n \times n$ identity matrix, then there exists at least one $2 \pi$ periodic solution of (1). The proof of this existence theorem, which was based on a slight modification of a theorem of C. L. Dolph [1] and the Brouwer fixed point theorem, did not imply uniqueness. The purpose of this note is to show that conditions far less restrictive than (2) and (3) imply that there can exist at most one $2 \pi$-periodic solution of (1). Our proof will be based on two very elementary abstract algebraic lemmas and the most basic properties of Fourier series.

LeMma 1. Let $V$ be a real vector space and let $H: V \times V \rightarrow R$ be a real valued symmetric bilinear form on $V$. If $V$ is the direct sum of subspaces $X$ and $Z$ such that $H$ is positive definite on $X$ and negative definite on $Z$, i.e.

Received by the editors June 21, 1971 and, in revised form, July 21, 1971.

AMS 1970 subject classifications. Primary 34C25, 15A63; Secondary 70D05.

Key words and phrases. Newtonian equations of motion, Sylvester's law of inertia, Parseval's formula. 
$H(x, x)>0$ if $x \in X, x \neq 0$, and $H(z, z)<0$ if $z \in Z, z \neq 0$, then $H$ is nondegenerate, i.e. if for some $w \in V, H(v, w)=0$ for all $v \in V$ then $w=0$.

Proof. If $V$ is finite dimensional the proof follows trivially from the existence of a basis of $V$ which is orthogonal with respect to $H$ and Sylvester's law of inertia. (See [3, Chapter XIV].) However, for the application we have in mind $V$ is infinite dimensional. Suppose $w \in V$ and $H(v, w)=0$ for all $v \in V$. Since $V$ is the direct sum of $X$ and $Z, w=x+z$ for some $x \in X$ and some $z \in Z$. Hence by the bilinearity and symmetry of $H$,

$$
\begin{aligned}
0 & =H(w, x-z)=H(x+z, x-z) \\
& =H(x, x)-H(x, z)+H(z, x)-H(z, z) \\
& =H(x, x)-H(z, z)
\end{aligned}
$$

so $H(x, x)=H(z, z)$. Since $H(x, x) \geqq 0$ and $H(z, z) \leqq 0, H(x, x)=$ $H(z, z)=0$. Thus by the conditions of the lemma $x=z=0$ so $w=0$. This proves the lemma.

LEMMA 2. Let $V$ be a vector space such that for subspaces $X$ and $Y$, $V=X \oplus Y$. If $Y$ is finite dimensional and $Z$ is a subspace of $V$ such that $X \cap Z=\{0\}$ and dimension $Y=$ dimension $Z$ then $V=X \oplus Z$.

Proof. Consider the sequence of linear maps

$$
Z \stackrel{i}{\longrightarrow} V \stackrel{j}{\longrightarrow} V \mid X
$$

where $i$ is inclusion and $j$ is the canonical projection of $V$ onto the quotient space $V / X$. Since the kernel of the composition $j \circ i$ is $X \cap Z=\{0\}$ the linear map $j \circ i$ is 1 to 1 . But since $V=X \oplus Y$ and $Y$ is finite dimensional,

$$
\text { dimensional } V / X=\text { dimensional } Y=\operatorname{dimensional} Z
$$

so $j \circ i$ is also onto. Let $\theta=(j \circ i)^{-1}$. From the commutativity of the triangle

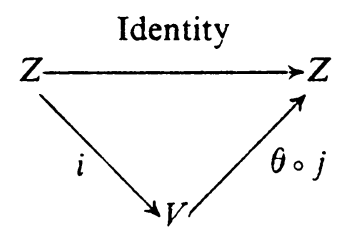

it follows from an elementary theorem on Abelian groups (see [2, p. 78] or $[3$, p. 44]) that

$$
V=\text { Image } i \oplus \text { Kernel } \theta \circ j=Z \oplus X .
$$

This proves the lemma. 
THEOREM 1. Let $Q$ be a real $n \times n$ symmetric matrix valued function with elements defined, continuous and $2 \pi$-periodic on the real line. Suppose there exist real constant symmetric matrices $A$ and $B$ such that

$$
A \leqq Q(t) \leqq B, \quad t \in(-\infty, \infty),
$$

and such that if $\lambda_{1} \leqq \lambda_{2} \leqq \cdots \leqq \lambda_{n}$ and $\mu_{1} \leqq \mu_{2} \leqq \cdots \leqq \mu_{n}$ denote the eigenvalues of $A$ and $B$ respectively then there exist integers $N_{k} \geqq 0, k=1,2, \cdots$, $n$, such that

$$
N_{k}^{2}<\lambda_{k} \leqq \mu_{k}<\left(N_{k}+1\right)^{2} .
$$

ASSERTION. There exists no nontrivial $2 \pi$-periodic solution of the vector differential equation

$$
u^{\prime \prime}+Q(t) w=0
$$

Proof. Let $\bar{a}_{1}, \bar{a}_{2}, \cdots, \bar{a}_{n}$ and $\bar{b}_{1}, \bar{b}_{2}, \cdots, \bar{b}_{n}$ be vectors such that

$$
\begin{array}{ll}
A \bar{a}_{k}=\lambda_{k} \bar{a}_{k}, & \left\langle\bar{a}_{k}, \bar{a}_{j}\right\rangle=\delta_{k j}, \\
B \bar{b}_{k}=\mu_{k} \bar{b}_{k}, & \left\langle\bar{b}_{k}, \bar{b}_{\jmath}\right\rangle=\delta_{k j},
\end{array}
$$

for $j, k=1, \cdots, n$ where $\langle$,$\rangle is the usual inner product on R^{n}$ and $\delta_{k j}=0$, $k \neq i, \delta_{k k}=1$. Let $V$ be the subspace of $C^{2}\left(R, R^{n}\right)$ consisting of the $2 \pi$ periodic members of the real vector space $C^{2}\left(R, R^{n}\right)$. We define subspaces $X$ and $Y$ of $V$ as follows:

1. $x \in X$ if

$$
\begin{gathered}
x(t)=\sum_{k=1}^{n} f_{k}(t) \tilde{b}_{k}, \\
f_{k}(t)=\sum_{r=N_{k}+1}^{\infty}\left(c_{k r} \cos r t+d_{k r} \sin r t\right)
\end{gathered}
$$

where $N_{k}$ is as in (5) and the series $f_{k}$ as well as series obtained by termwise differentiation of (10) converges uniformly on $R$;

II. $y \in Y$ if

$$
\begin{gathered}
y(t)=\sum_{k=1}^{n} g_{k}(t) \tilde{b}_{k}, \\
g_{k}(t)=c_{k 0}+\sum_{r=1}^{N_{k}}\left(c_{k r} \cos r t+d_{k r} \sin r t\right) .
\end{gathered}
$$

Clearly $V=X \oplus Y$. Indeed if $v \in V, v(t)=\sum_{k=1}^{n}\left\langle v(t), \bar{b}_{k}\right\rangle \bar{b}_{k}$. Since $\left\langle v(t), \bar{b}_{k}\right\rangle$ is a twice differentiable $2 \pi$-periodic function it follows from the theory of Fourier series that $\left\langle v(t), \tilde{b}_{k}\right\rangle=g_{k}(t)+f_{k}(t)$ where $f_{k}$ and $g_{k}$ are uniquely determined and have the forms given by (10) and (12) respectively. 
We define a real bilinear symmetric form $H$ on $V$ as follows:

If $u \in V, v \in V$,

$$
H(u, v)=\int_{0}^{2 \pi}\left(\left\langle u^{\prime}(t), v^{\prime}(t)\right\rangle-\langle u(t), Q(t) v(t)\rangle\right) d t .
$$

We assert that $H$ is positive definite on $X$. Indeed let $x \in X$. From (4) we see that

$$
H(x, x) \geqq \int_{0}^{2 \pi}\left(\left\langle x^{\prime}(t), x^{\prime}(t)\right\rangle-\langle x(t), B x(t)\rangle\right) d t .
$$

But if $x$ is represented in the form (9) it follows from (8), (10) and Parseval's formula that

$$
\begin{aligned}
\int_{0}^{2 \pi}\left(\left\langle x^{\prime}(t), x^{\prime}(t)\right\rangle-\langle x(t), B x(t)\rangle\right) d t & =\sum_{k=1}^{n} \int_{0}^{2 \pi} f_{k}^{\prime}(t)^{2} d t-\sum_{k=1}^{n} \mu_{k} \int_{0}^{2 \pi} f_{k}(t)^{2} d t \\
& =\sum_{k=1}^{n} \sum_{r=V_{k}+1}^{\infty} \pi\left(r^{2}-\mu_{k}\right)\left(c_{k r}^{2}+d_{k r}^{2}\right) \geqq 0
\end{aligned}
$$

with equality only if $c_{k r}=d_{k r}=0$ for $k=1, \cdots, n$ and $r \geqq N_{k}+1$ for, according to condition (5), $r^{2}>\mu_{k}$ if $r \geqq N_{k}+1$. Hence from (14) we see at once that $H(x, x)>0$ if $x \in X$ and $x \neq 0$.

We now define another subspace $Z$ of $V$ as follows:

III. $z \in Z$ if

$$
\begin{gathered}
z(t)=\sum_{k=1}^{n} h_{k}(t) \vec{a}_{k}, \\
h_{k}(t)=p_{k 0}+\sum_{r=1}^{N_{k}}\left(p_{k r} \cos r t+q_{k r} \sin r t\right),
\end{gathered}
$$

where $\bar{a}_{k}$ is defined by (7).

We assert that $H$ is negative definite on $Z$ Indeed let $z$ be represented in the form (15). From (13), (4), (7), and (16) it follows that

$$
\begin{aligned}
H(z, z) & \leqq \int_{0}^{2 \pi}\left(\left\langle z^{\prime}(t), z^{\prime}(t)\right\rangle-\langle z(t), A z(t)\rangle\right) d t \\
& =\sum_{k=1}^{n} \int_{0}^{2 \pi} h_{k}^{\prime}(t)^{2} d t-\sum_{k=1}^{n} \lambda_{k} \int_{0}^{2 \pi} h_{k}(t)^{2} d t \\
& =\sum_{k=1}^{n} \sum_{r=1}^{N_{k}} \pi r^{2}\left(p_{k r}^{2}+q_{k r}^{2}\right)-\sum_{k=1}^{n} \lambda_{k}\left(2 \pi p_{k 0}^{2}+\sum_{r=1}^{\lambda_{k}} \pi\left(p_{k r}^{2}+q_{k r}^{2}\right)\right) \leqq 0
\end{aligned}
$$

with equality only if $p_{k r}=0$ for $k=1, \cdots, n, r=0, \cdots, N$ and $q_{k r}=0$ for $k=1, \cdots, n, r=1, \cdots, N_{k}$ for, by (5), $r^{2}<\lambda_{k}$ if $r \leqq N_{k}$. This shows $H$ is negative definite on $Z$. 
Since $H$ is positive definite on $X$ and negative definite on $Z$ we see that $X \cap Z=\{0\}$. Moreover by comparing (11) and (12) with (15) and (16) it is readily seen that

$$
\text { dimension } Y=\operatorname{dimension} Z=\sum_{k=1}^{n}\left(2 N_{k}+1\right) \text {. }
$$

Thus since it was shown above that $V=X \oplus Y$ it follows by application of Lemma 2 that $V=X \oplus Z$. We may therefore apply Lemma 1 to conclude that if $H(v, w)=0$ for all $v \in V$ then $w=0$.

To finish the proof of Theorem 1 suppose $w \in V$ satisfies (6). If $v \in V$ is arbitrary then

$$
\int_{0}^{2 \pi}\left(-\left\langle v(t), w^{\prime \prime}(t)\right\rangle-\langle v(t), Q(t) w(t)\rangle\right) d t=0 .
$$

Integrating the first term by parts and noticing that the boundary terms cancel by periodicity of $v$ and $w$ we find that

$$
H(v, w)=\int_{0}^{2 \pi}\left(\left\langle v^{\prime}(t), w^{\prime}(t)\right\rangle-\langle v(t), Q(t) w(t)\rangle\right) d t=0 .
$$

Since $v \in V$ is arbitrary, $w(t)=0$ for all $t$. This proves Theorem 1 .

The main goal of this note is the following:

TheORem 2. Let $G \in C^{2}\left(R^{n}, R\right)$. If there exist two constant symmetric matrices $A$ and $B$ satisfying the conditions of Theorem 1 such that, for all $a \in R^{n}$,

$$
A \leqq\left(\partial^{2} G(a) / \partial x_{i} \partial x_{j}\right) \leqq B
$$

then there can exist at most one $2 \pi$-periodic solution of (1).

Proof. Suppose $\phi_{1}(t)$ and $\phi_{2}(t)$ are $2 \pi$-periodic solutions of (1). By the integral mean-value theorem for vector functions (see for example $[4$, p. 103])

$$
\operatorname{grad} G\left(\phi_{1}(t)\right)-\operatorname{grad} G\left(\phi_{2}(t)\right)=Q(t)\left(\phi_{1}(t)-\phi_{2}(t)\right)
$$

where

$$
Q(t)=\int_{0}^{1}\left(\frac{\partial^{2} G}{\partial x_{i} \partial x_{j}}\left(\phi_{2}(t)+s\left(\phi_{2}(t)-\phi_{1}(t)\right)\right)\right) d s .
$$

Clearly $A \leqq Q(t) \leqq B$ for all $t$. If $w=\phi_{1}-\phi_{2}$ then $w$ is a $2 \pi$-periodic solution of (6) so, by Theorem $1, w^{\prime}(t)=0$ for all $t$. This proves the theorem.

Remark. Úsing completely different methods Professor Shair Ahmad and the author have recently proved that the conditions of Theorem 2 
imply existence of a $2 \pi$-periodic solution of (1). However, the proof is lengthy and involves techniques less elementary than the ones used here.

\section{REFERENCES}

1. C. L. Dolph, Nonlinear integral equations of the Hammerstein type, Trans. Amer. Math. Soc. 66 (1949), 289-307. MR 11, 367.

2. S. T. Hu, Elements of modern algebra, Holden-Day, San Francisco, Calif., 1965. MR 31 \#3444.

3. S. Lang, Algebra, Addison-Wesley, Reading, Mass., 1965. MR 33 \#5416.

4. —-, Analysis. II, Addison-Wesley, Reading, Mass., 1969.

5. A. C. Lazer and D. A. Sánchez, On periodically perturbed conservative systems, Michigan Math. J. 16 (1969), 193-200. MR 39 \#7212.

Department of Mathematics, University of Cincinnati, Cincinnati, Oho 45221 